\title{
Investigating microscopic seepage characteristics and fracture effectiveness of tight sandstones: a digital core approach
}

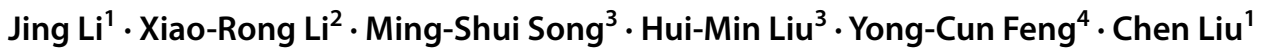

Received: 2 December 2019 / Published online: 28 May 2020

(c) The Author(s) 2020

\begin{abstract}
Microscopic seepage characteristics are critical for the evaluation of tight sandstone reservoirs. In this study, a digital core approach integrating microscopic seepage simulation and CT scanning was developed to characterize microscopic seepage and fracture effectiveness (the ratio of micro-fractures that contributes to fluid flow) of tight sandstones. Numerical simulations were carried out for characterizations of tight sandstones. The results show that the axial permeability of the investigated cylindrical tight sandstone from Junggar Basin in China is $0.460 \mu \mathrm{m}^{2}$, while the radial permeability is $0.3723 \mu \mathrm{m}^{2}$, and the axial and radial effective fracture ratios are 0.4387 and 0.4806 , respectively, indicating that cracks are not fully developed and the connectivity between micro-cracks is poor. Directional permeability that is difficult to measure by laboratory experiments can be obtained readily using the proposed method in this paper. The results provide important information for improving the exploration and development of tight sandstone reservoirs.
\end{abstract}

Keywords Tight sandstone $\cdot$ Digital core approach $\cdot$ CT scanning $\cdot$ Microscopic seepage characteristics $\cdot$ Fracture effectiveness

\section{Introduction}

Seepage characteristics of reservoir rocks are important information in exploration and development of tight oil and gas reservoirs. Most existing investigations on seepage characteristics are based on macroscopic scale studies (Li et al. 2019a, b; Lv et al. 2019; Zhang et al. 2019). Because the pore size of tight sandstone is relatively small

Edited by Yan-Hua Sun

$\triangle$ Jing Li

lijing0681@163.com

$\triangle$ Xiao-Rong Li

xiaorongli@cup.edu.cn

1 College of Pipeline and Civil Engineering, China University of Petroleum (East China), Qingdao 266580, Shandong, China

2 College of Safety and Ocean Engineering, China University of Petroleum (Beijing), Beijing 102220, China

3 Sinopec Shengli Oilfield Company, Dongying 257000, Shandong, China

4 College of Petroleum Engineering, China University of Petroleum (Beijing), Beijing 102220, China (at micron level), it is necessary to carry out microscopic scale study of seepage characteristics of tight sandstone reservoirs (Babchin and Nasr 2006; Li et al. 2019a, b; Ren et al. 2018; Yin et al. 2019; Wang et al. 2020). The study of micro-seepage of reservoir rocks is usually through restoring the micro-pore structures of the rock in numerical models. On this basis, heterogeneous seepage models were developed and seepage characteristics of the rocks were analyzed through numerical simulations. Digital core technology has been extensively used to characterize the heterogeneity of rocks in the aspects of solid mechanics, mesoscopic mechanics, and rock damage (Lebourg et al. 2004; Nishank et al. 2019; Sadegh and Pejman 2019; Shan and Lai 2019). Some researchers have also used the digital core technology to study microscopic percolation mechanism of rocks. However, these applications of digital core technology are mostly based on random mathematical models (Song and Zhou 2019; Starnoni et al. 2017; Sun et al. 2019; Wang et al. 2019a, b).

Effectiveness of fractures can reflect the permeability of rocks (Zhang et al. 2013; Zhang and Mack 2017). Effective fracture ratio directly affects the production performance of oil and gas reservoirs, so it is an important index for evaluating oil and gas reservoirs. To date, the studies of fracture 
effectiveness are mostly based on qualitative analysis of well logging information (Tang et al. 2017), fracture genesis, and influential factors (An et al. 2016), while quantitative evaluation of fracture effectiveness is rare (Zhao et al. 2017). Therefore, this paper proposes a digital core approach to study heterogeneous seepage characteristics of tight sandstones by incorporating micro-CT scanning results into a numerical fluid flow model. Next, micro-seepage characteristics of tight sandstones are investigated using numerical simulations. Furthermore, fracture effectiveness is quantitatively determined based on the simulation results of micro-seepage.

\section{Micro-CT scanning}

Tight sandstone samples taken from the Jurassic Toutunhe Formation in Junggar Basin, China, were scanned with a MicroCT scanner-MicroXCT-200. A total of 5 sandstone samples were used for the experiments. A total number of $10082 \mathrm{D}$ grayscale images with $1024 \times 1024$ pixels and a reconstruction resolution of $2.3 \mu \mathrm{m}$ (each pixel in the image corresponds to an actual length of $2.3 \mu \mathrm{m}$ ) were obtained for each rock sample. After omitting some invalid images at the beginning and the end of the scanning process, each sample had about 950 valid 2D grayscale images. Figure 1a shows an example of the 2D images. The dark black areas represent rock pores and micro-fissures, while the bright white areas are high-density mineral components of the rock (e.g., pyrite, calcite, etc.), and the intermediate gray color areas between dark black and bright white are feldspar and clay minerals.

By superimposing the effective 2D grayscale images of each sample, a 3D grayscale image of the rock sample can be obtained. An example is shown in Fig. 1b. In the following, a numerical fluid seepage model for the heterogeneous rock samples will be developed using the 3D image to simulate micro-seepage behavior of the rock and to evaluate fracture effectiveness.

\section{Heterogeneous model of the tight sandstone}

Using 3D grayscale CT scan images of the rock samples, 2D numerical models were established for various vertical cross section planes intersecting the axis of the core samples. To better capture the microscale seepage characteristics of the tight sandstone samples, a total number of 12 vertical cross section planes were selected for each sample with a $15^{\circ}$ apart angle between each cross section planes. Figure 2 shows the selection of these planes, i.e., planes of $0^{\circ}-180^{\circ}, 15^{\circ}-195^{\circ}, 30^{\circ}-210^{\circ}, 45^{\circ}-225^{\circ}, 60^{\circ}-240^{\circ}$, $75^{\circ}-255^{\circ}, 90^{\circ}-270^{\circ}, 105^{\circ}-285^{\circ}, 120^{\circ}-300^{\circ}, 135^{\circ}-315^{\circ}$, $150^{\circ}-330^{\circ}$, and $165^{\circ}-345^{\circ}$. Figure 3 shows an example of the cross section plane of $0^{\circ}-180^{\circ}$, with $x$ and $y$ directions corresponding to the axial and radial directions of the core sample, respectively.

Since different grayscale values (intensity) in the grayscale image correspond to different medium regions, the micro-cracks, pores and rock skeletons of each cross section can be distinguished by threshold segmentation of the gray value (the threshold value is 58). The black regions in the images correspond to cracks and pores. The distributions of the cracks are identified and imported into a 2D pore structure model in the multiphysics modeling softwareCOMSOL. The crack width is in the range of 2.3 to $9.2 \mu \mathrm{m}$ interpreted from the images. Figure 4 shows the identified micro-crack distribution image of the $0^{\circ}-180^{\circ}$ plane and Fig. 5 shows the corresponding crack distribution in the pore structure model in COMSOL.

The fluid used in the simulations is assumed to be water with a density of $1000 \mathrm{~kg} / \mathrm{m}^{3}$, a dynamic viscosity of $0.001 \mathrm{~Pa} / \mathrm{s}$, and a bulk modulus of $2.18 \mathrm{GPa}$. The fluid
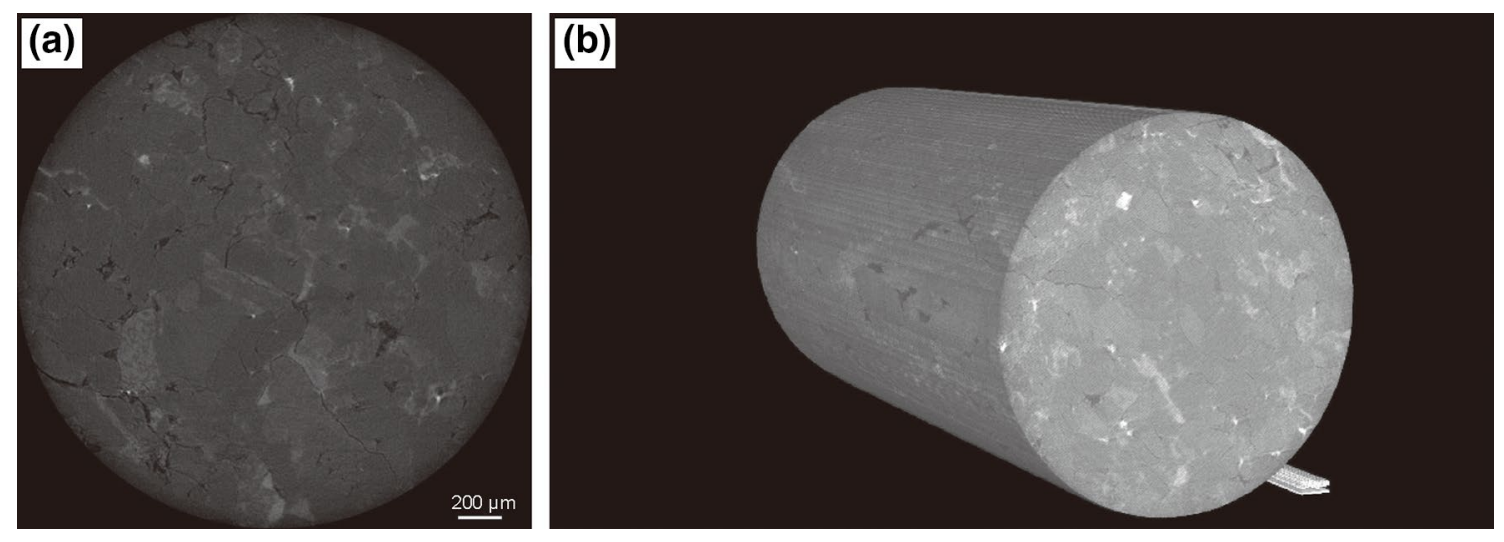

Fig. 1 2D grayscale CT scan image of a tight sandstone sample (a) and 3D grayscale image of the sample (b) 


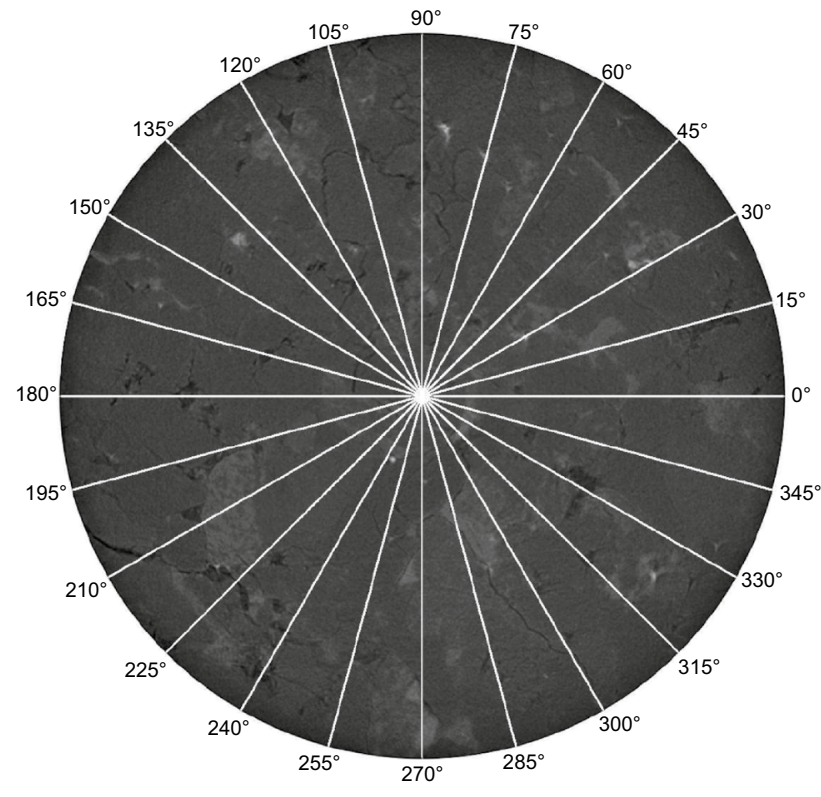

Fig. 2 Directions of the selected cross section planes

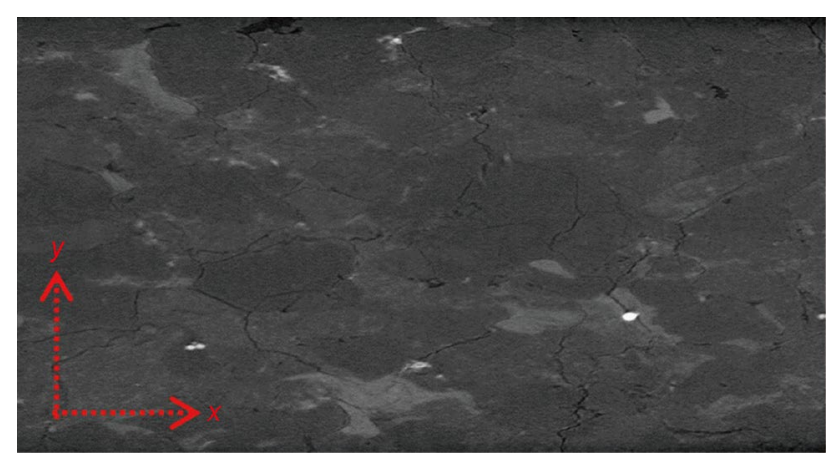

Fig. 3 A cross section plane along the $0^{\circ}-180^{\circ}$ direction in Fig. 2

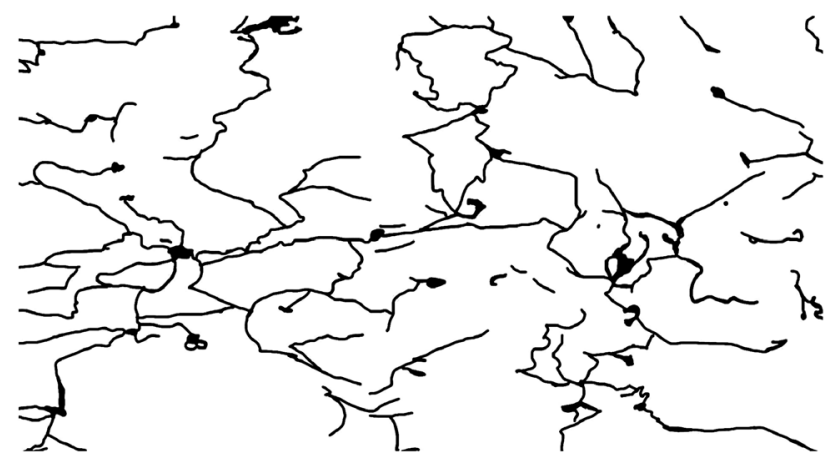

Fig. 4 Micro-crack distribution in the $0^{\circ}-180^{\circ}$ plane

can flow within micro-cracks and pores in the rock. Fluid is injected separately into the cross section in the $x$ and $y$ directions to study the axial and radial seepage characteristics of

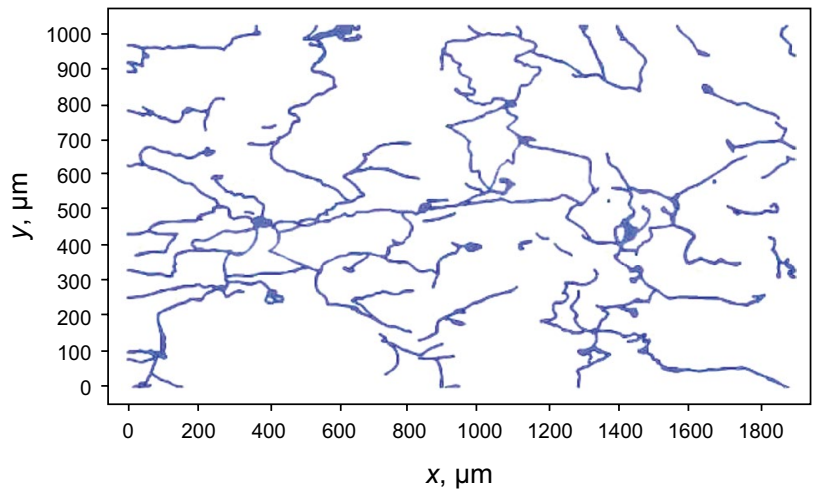

Fig. 5 Micro-crack distribution in the pore structure model in COMSOL

the core samples. In the $x$ direction (i.e., axial direction of the core), the fluid injection pressure at the left boundary is $20 \mathrm{~Pa}$ and the outlet pressure at the right boundary is $0 \mathrm{~Pa}$. For fluid injection along the $y$ direction (i.e., radial direction of the core), the injection pressure at the bottom side of the $2 \mathrm{D}$ cross section plane is $10 \mathrm{~Pa}$ and the outlet pressure at the top side of the plane is $0 \mathrm{~Pa}$. It should be noted that, because of the high scanning precision, micro-cracks and pores are basically captured. While fluid leakage and matrix adsorption eventually result in fluid entering the matrix, it is very difficult for the fluid to penetrate to the boundary of the model and the influence on permeability is negligible. Therefore, it is assumed that the fluid can only flow continuously in the micro-cracks and pores, and the boundary between the micro-cracks and matrix is set as impermeable boundary.

\section{Analysis of microscopic seepage characteristics of tight sandstones}

\subsection{Fluid flow model}

To date, there are two main modeling approaches for the simulation of microscopic seepage of rock using digital core technology - the pore network model and the digital core model (Mutailipu et al. 2017). The pore network model replaces complicated pores and throats in the rock with capillaries of different diameters, thus transforming fluid flow in complex pore structure into flow in regular capillaries. It is obvious that considerable simplification of pore/throat structures of the rock should be made in the pore network model. Conversely, the digital core model is based on the real pore/throat/crack structure of the rock to study the seepage characteristics, which overcomes many shortcomings of the pore network model. Therefore, digital core modeling 
technique is used in this study to characterize the microseepage behavior of tight sandstones.

There are two main numerical fluid mechanics methods for simulating microscopic fluid seepage in digital core models - the Lattice Boltzmann method (LBM) based on discrete medium assumption and the compute fluid dynamic (CFD) method based on continuous medium assumption. In this paper, the CFD method and Navier-Stokes equations are used to model fluid seepage in the micro-structures of tight sandstones.

Navier-Stokes equations are equations of motion that describe the momentum conservation of fluids, expressed as:

$$
\left.\begin{array}{l}
\frac{\partial \rho}{\partial t}+\nabla \cdot(\rho u)=0 \\
\frac{\partial(\rho u)}{\partial t}+\nabla \cdot(\rho u u)=\nabla \sigma \\
\frac{\partial(\rho e)}{\partial t}+\nabla \cdot(\rho e u)=\sigma \cdot \nabla u-\nabla q
\end{array}\right\}
$$

where $\rho$ is the fluid density; $u$ is the fluid velocity; $e$ is the internal energy of the fluid; $q$ is the heat flux; $\sigma$ is the stress tensor; $\nabla$ is Laplace operator; $t$ is time.

The vector expressions of Navier-Stokes equations are as follows:

$\rho \frac{\partial u}{\partial t}+\rho(u \cdot \nabla) u=\nabla \cdot\left[-p+\mu\left(\nabla u+(\nabla u)^{T}\right)\right]+F$

$\rho \nabla u=0$

where $F$ is the force acting on the fluid; $p$ is the pressure.

\subsection{Simulation results of microscopic seepage in tight sandstones}

Simulations of microscopic seepage along $x$ and $y$ directions of the twelve cross section planes in Fig. 2 were carried out.
The distributions of fluid flow velocity and fluid pressure in the micro-cracks of in the $x$ (corresponding to the axial direction of core cylinder) and $y$ directions (corresponding to the radial direction of core cylinder) were obtained. To illustrate the capability of the model, simulation results of the $0^{\circ}-180^{\circ}, 45^{\circ}-225^{\circ}, 90^{\circ}-270^{\circ}, 135^{\circ}-315^{\circ}$ planes are shown through Figs. 6, 7, 8, 9, 10, 11, 12 and 13.

From fluid pressure and flow velocity fields shown in these figures, it can be observed that:

- Along fluid flow paths, larger pressure drop and larger flow velocity appear near the turning points of the flow paths.

- Many micro-cracks have essentially zero fluid flow velocity, indicating the effectiveness of these micro-cracks is poor.

\subsection{Calculation of permeability based on the simulation results}

Based on the simulation results of fluid seepage, the distribution of the flow velocity along the fluid exit boundary can be obtained. For example, Fig. 14 shows the fluid discharge velocity at the $x$-exiting boundary of the $0^{\circ}-180^{\circ}$ plane. The flow rate can, therefore, be obtained by integrating the flow velocity along the boundary. According to Darcy's law, the permeability at this direction can be expressed as:

$K=\frac{Q \mu L}{A \Delta P}$

where $Q$ is the flow rate; $A$ is the cross section area, 1896 $\mu \mathrm{m}^{2}$ in the $x$ direction and $1024 \mu \mathrm{m}^{2}$ in the $y$ direction; $L$ is the length along the flow direction, $1896 \mu \mathrm{m}$ in the $x$ direction and $1024 \mu \mathrm{m}$ in the $y$ direction; $\Delta P$ is the pressure difference, $10 \mathrm{~Pa}$; $\mu$ is the fluid viscosity, $0.001 \mathrm{~Pa} \cdot \mathrm{s}$. The only variable is the fluid flow rate $Q$, which can be calculated by
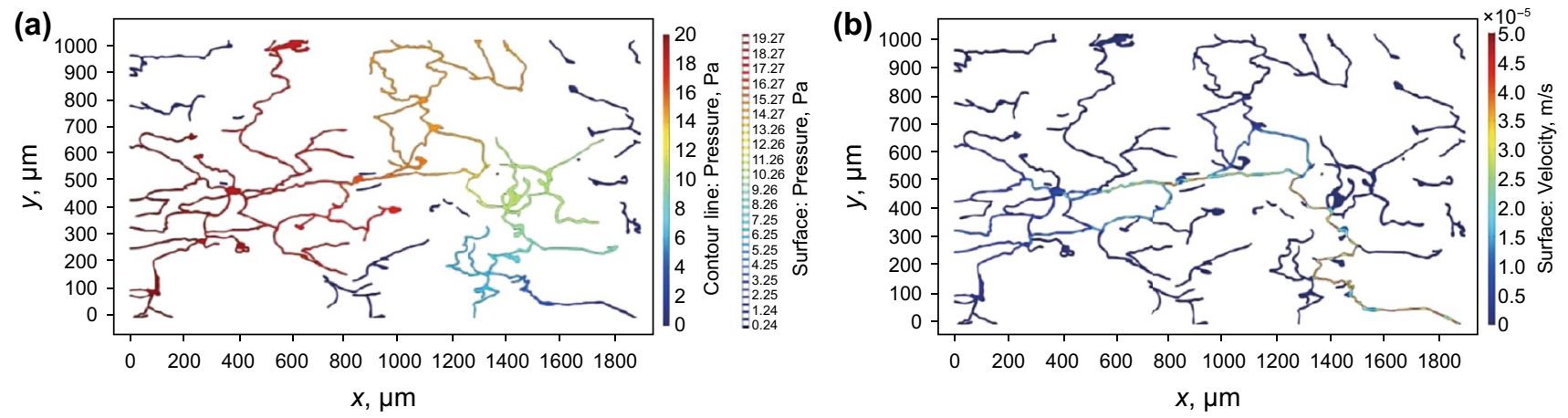

Fig. 6 Fluid pressure field (a) and fluid velocity field (b) for $x$ direction flow simulation in the $0^{\circ}-180^{\circ}$ cross section plane 

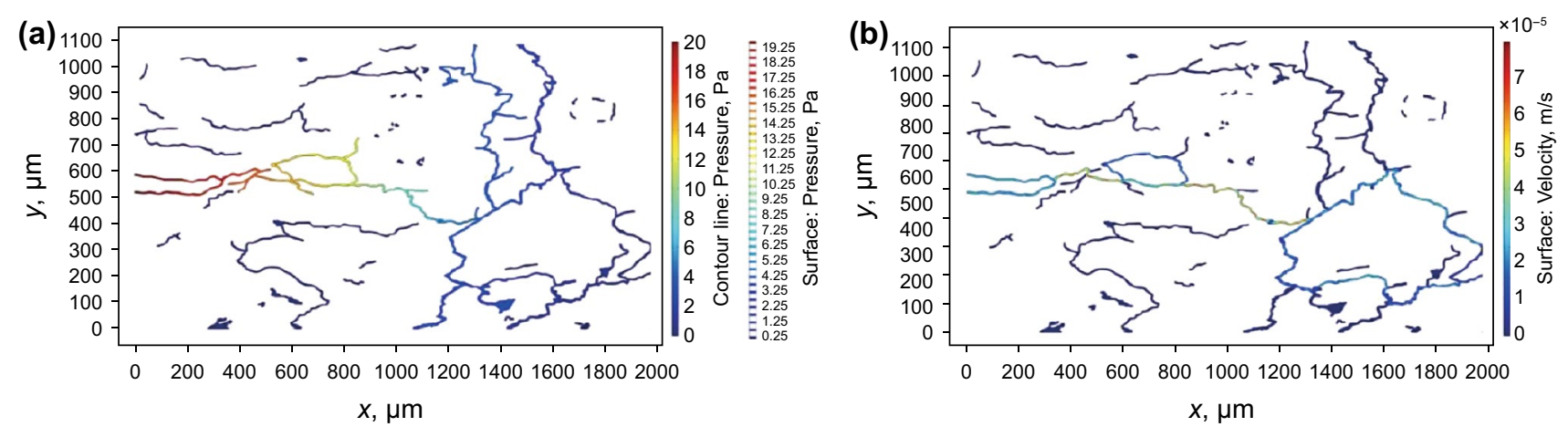

Fig. 7 Fluid pressure field (a) and fluid velocity field (b) for $x$ direction flow simulation in the $45^{\circ}-225^{\circ}$ cross section plane
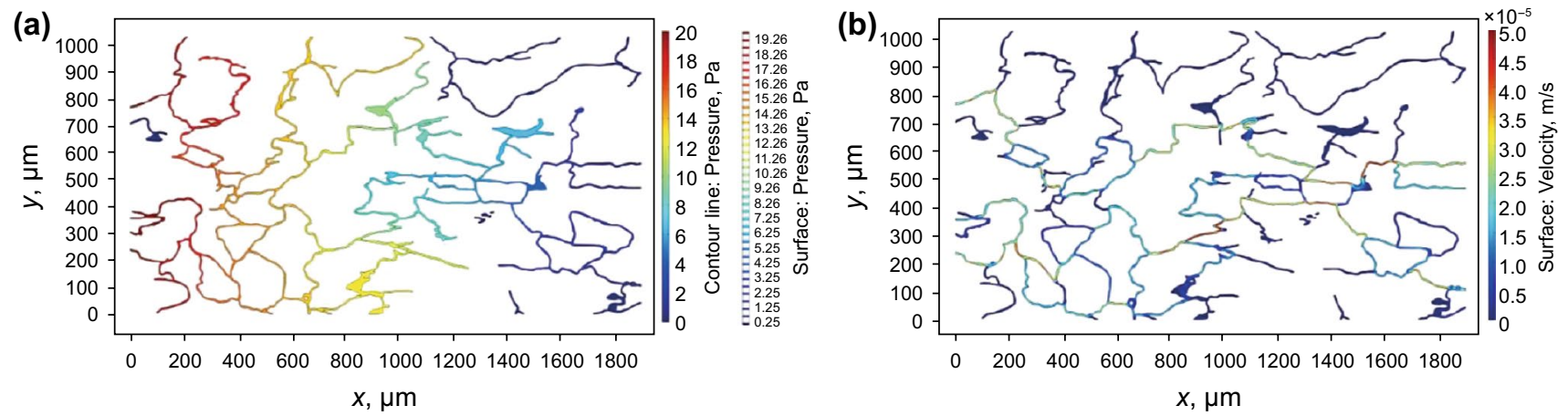

Fig. 8 Fluid pressure field (a) and fluid velocity field (b) for $x$ direction flow simulation in the $90^{\circ}-270^{\circ}$ cross section plane
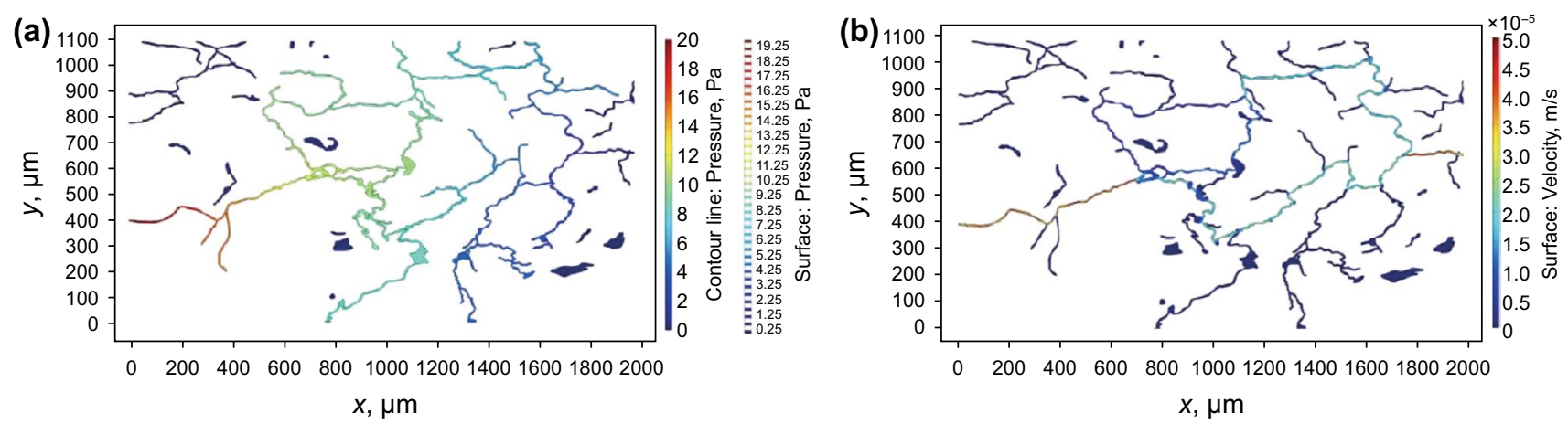

Fig. 9 Fluid pressure field (a) and fluid velocity field (b) for $x$ direction flow simulation in the $135^{\circ}-315^{\circ}$ cross section plane

integrating the flow velocity along the outlet boundary (as shown in Fig. 14).

The outlet flow rates and corresponding permeability in the $x$ direction (the axial direction of the core) of 12 cross section planes are summarized in Table 1. It can be found that the permeability of different planes vary greatly. The main reason for these differences is the strong heterogeneity of cracks and pores in the tight sandstone rock. The average permeability from numerical simulation is slightly higher than the measured permeability. The reasons for this small discrepancy could be due to the pore blockage by water-sensitive minerals in the laboratory measurement, which was not considered in the simulation; or due to the limitation of micro-CT scanning accuracy (identifications of some very tiny micro-cracks were poor). 

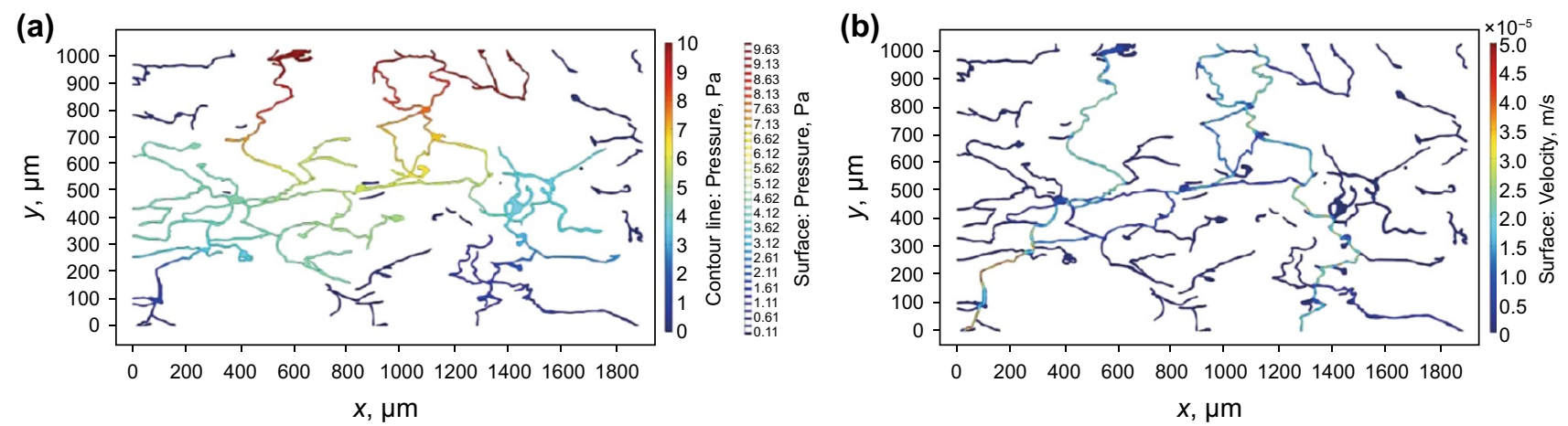

Fig. 10 Fluid pressure field (a) and fluid velocity field (b) for $y$ direction flow simulation in the $0^{\circ}-180^{\circ}$ cross section plane
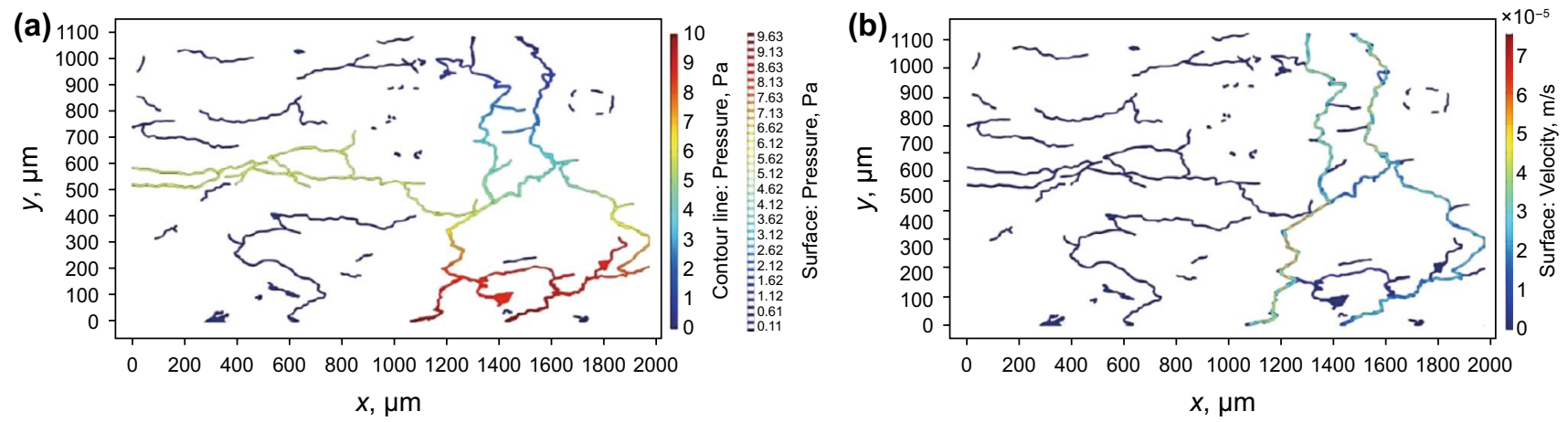

Fig. 11 Fluid pressure field (a) and fluid velocity field (b) for $y$ direction flow simulation in the $45^{\circ}-225^{\circ}$ cross section plane
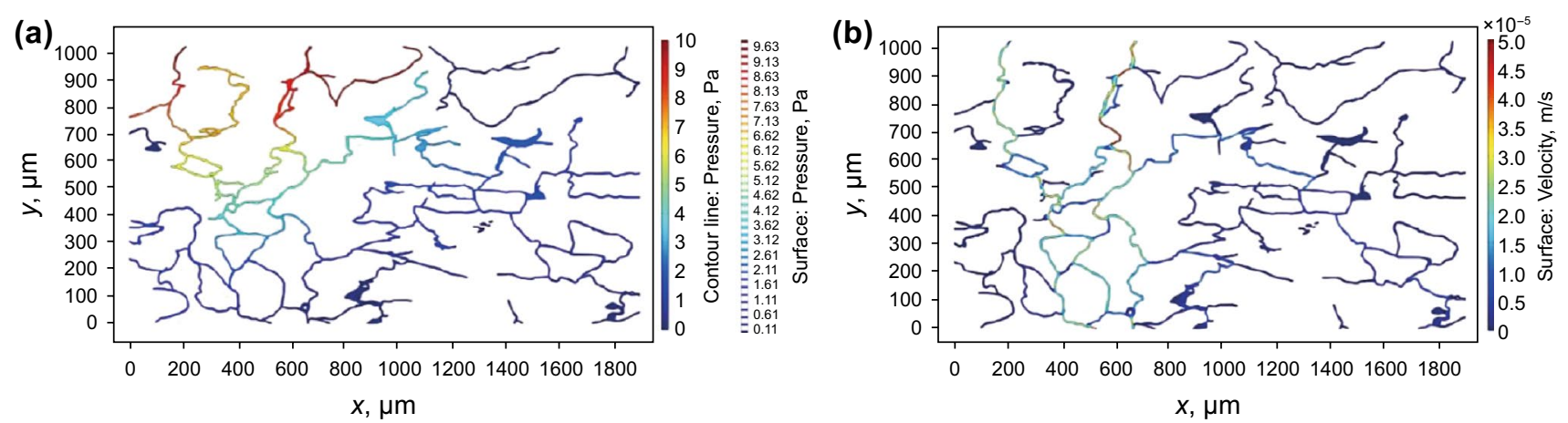

Fig. 12 Fluid pressure field (a) and fluid velocity field (b) for $y$ direction flow simulation in the $90^{\circ}-270^{\circ}$ cross section plane

Similarly, the permeability along the $y$ direction (the radial direction of the core) of the 12 cross section planes was obtained through numerical simulations. The results are shown in Table 2. It can be seen that the radial permeability of core is quite different from the axial permeability, indicating strong heterogeneity of the micro-structure of the tight sandstone sample.
In conventional laboratory tests, the axial permeability is usually measured. However, it is not easy to directly measure the radial permeability. In order to experimentally measure directional permeability, rock samples cored at different directions must be prepared, which is usually time-consuming and expensive, or even not possible for some highly fractured rocks. The digital core approach using micro-CT 

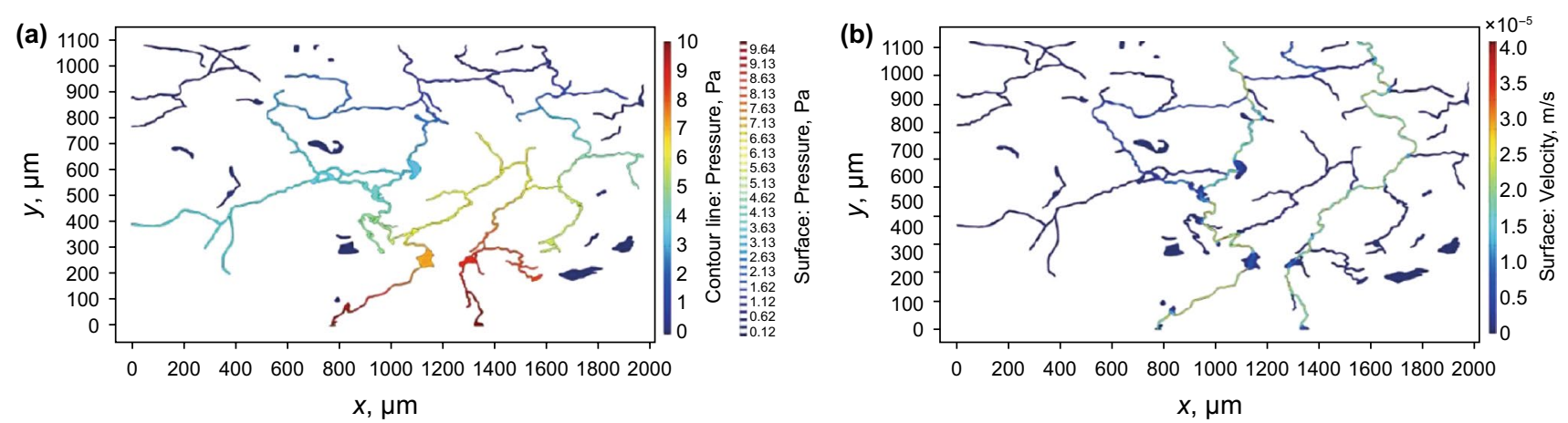

Fig. 13 Fluid pressure field (a) and fluid velocity field (b) for $y$ direction flow simulation in the $135^{\circ}-315^{\circ}$ cross section plane

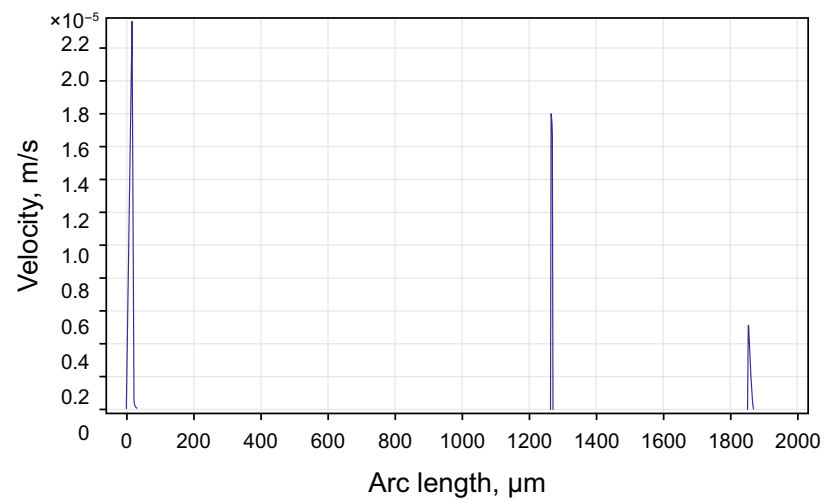

Fig. 14 Fluid discharge velocity at the $x$-exiting boundary of the $0^{\circ}-180^{\circ}$ cross section plane

scanning and numerical simulation proposed in this paper can overcome these limitations and obtain permeability at any interested directions without complex coring process.

\section{Quantitative evaluation of fracture effectiveness}

According to the simulation results of fluid flow velocity, it can be observed that fluid velocity in many micro-cracks is essentially zero. Therefore, this part of micro-fractures can be regarded as ineffective fractures. A reason for this is the incomplete development of micro-fractures in the tight sandstone that results in poor connectivity among them. Therefore, the capability to quantitatively evaluate the effectiveness of fracture (i.e., the ratio of micro-fractures that contributes to fluid flow) is vital for the evaluation of tight sand reservoirs.

In this section, the effectiveness of micro-fractures is statistically analyzed based on the simulation results of micro-seepage. Specific analysis procedures of the analysis are as follows:

Table 1 Simulation results of the axial permeability of the core sample

\begin{tabular}{llllll}
\hline Section direction & Export flow, $\mathrm{m}^{2} / \mathrm{s}$ & $\begin{array}{l}\text { Simulated per- } \\
\text { meability, } 10^{-3} \\
\mu \mathrm{m}^{2}\end{array}$ & $\begin{array}{l}\text { Average permeability, } \mu^{-3} \\
\mu \mathrm{m}^{2}\end{array}$ & $\begin{array}{l}\text { Standard deviation, } 10^{-3} \\
\mu \mathrm{m}^{2}\end{array}$ & $\begin{array}{l}\text { Measured permeability, } 10^{-3} \\
\mu \mathrm{m}^{2}\end{array}$ \\
\hline $0^{\circ}-180^{\circ}$ & $2.2353 \times 10^{-10}$ & 0.5405 & 0.460 & 0.185 & 0.394 \\
$15^{\circ}-195^{\circ}$ & $1.9654 \times 10^{-10}$ & 0.4531 & & \\
$30^{\circ}-210^{\circ}$ & $1.1724 \times 10^{-10}$ & 0.2691 & & \\
$45^{\circ}-225^{\circ}$ & $2.9295 \times 10^{-10}$ & 0.6739 & & \\
$60^{\circ}-240^{\circ}$ & $2.0860 \times 10^{-10}$ & 0.4807 & & \\
$75^{\circ}-255^{\circ}$ & $2.7741 \times 10^{-10}$ & 0.6371 & & \\
$90^{\circ}-270^{\circ}$ & $3.7625 \times 10^{-10}$ & 0.8648 & & \\
$105^{\circ}-285^{\circ}$ & $1.1959 \times 10^{-10}$ & 0.2760 & & \\
$120^{\circ}-300^{\circ}$ & $1.4274 \times 10^{-10}$ & 0.3289 & & \\
$135^{\circ}-315^{\circ}$ & $2.0389 \times 10^{-10}$ & 0.4692 & & \\
$150^{\circ}-330^{\circ}$ & $1.0646 \times 10^{-10}$ & 0.2438 & & \\
$165^{\circ}-345^{\circ}$ & $1.2393 \times 10^{-10}$ & 0.2852 & & \\
\hline
\end{tabular}


Table 2 Simulation results of the radial permeability of the core sample

\begin{tabular}{|c|c|c|c|c|}
\hline $\begin{array}{l}\text { Section direction, } \\
\text { degree }\end{array}$ & Export flow, $\mathrm{m}^{2} / \mathrm{s}$ & $\begin{array}{l}\text { Calculated permeability, } \\
10^{-3} \mu \mathrm{m}^{2}\end{array}$ & Average permeability, $10^{-3} \mu \mathrm{m}^{2}$ & Standard deviation, $10^{-3} \mu \mathrm{m}^{2}$ \\
\hline $0-180$ & $3.5289 \times 10^{-10}$ & 0.4058 & 0.3723 & 0.1399 \\
\hline 15-195 & $2.5222 \times 10^{-10}$ & 0.2901 & & \\
\hline $30-210$ & $2.5710 \times 10^{-10}$ & 0.2957 & & \\
\hline $45-225$ & $5.2981 \times 10^{-10}$ & 0.6093 & & \\
\hline $60-240$ & $3.5336 \times 10^{-10}$ & 0.4064 & & \\
\hline $75-255$ & $2.2184 \times 10^{-10}$ & 0.2551 & & \\
\hline $90-270$ & $4.5987 \times 10^{-10}$ & 0.5703 & & \\
\hline $105-285$ & $1.2377 \times 10^{-10}$ & 0.1423 & & \\
\hline $120-300$ & $2.1425 \times 10^{-10}$ & 0.2464 & & \\
\hline $135-315$ & $2.5598 \times 10^{-10}$ & 0.2944 & & \\
\hline $150-330$ & $4.8811 \times 10^{-10}$ & 0.5613 & & \\
\hline $165-345$ & $3.3996 \times 10^{-10}$ & 0.3910 & & \\
\hline
\end{tabular}

(1) Remove cracks with 'zero' flow velocity (velocity less than $1 \times 10^{-7} \mathrm{~m} / \mathrm{s}$ ) in the fluid velocity images. In this paper, the 'nonfunctional' cracks are essentially the isolated cracks (cracks not connected with other cracks), or some partially connected cracks that are not belonging to the main channels for seepage and fluid within them cannot flow freely. These cracks have no contribution to permeability calculation. The selection of $10^{-7} \mathrm{~m} / \mathrm{s}$ as a criterion is because, in the simulated cloud maps of flow velocity, the velocities in the "nonfunctional' cracks are generally below $10^{-7} \mathrm{~m} / \mathrm{s}$.

(2) Transfer the fluid flow images before and after removing zero-velocity fractures into grayscale images, and then carry out the processing of image segmentation.

(3) Count the number of pixels of all the fracture area $\left(N_{\text {all }}\right)$ and that of the fracture area with nonzero flow velocity $\left(N_{\text {eff }}\right)$.

(4) Calculate fracture effectiveness $n=N_{\text {eff }} / N_{\text {all }}$.

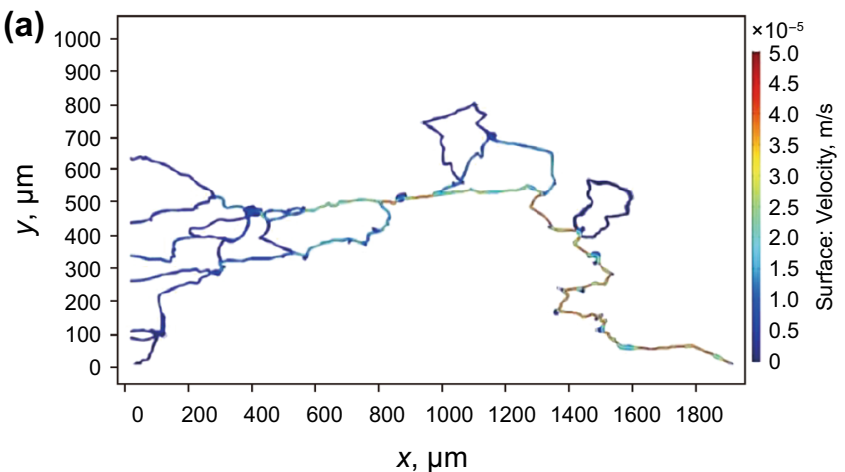

Figures 15 and 16 show the effective cracks in the $x$ and $y$ directions in the $0-180^{\circ}$ and $90-270^{\circ}$ cross section planes, respectively. A MATLAB program was developed to process the images of seepage simulation results. Tables 3 and 4 summarize the image processing results and calculated fracture effectiveness in the $x$ direction (axial direction of the core) and $y$ direction (radial direction of the core) of the 12 cross section planes, respectively. The axial and radial fracture effectiveness are 0.4387 and 0.4806 , respectively, indicating the overall fracture effectiveness is low, which is unfavorable for the effective exploitation of oil and gas reservoirs. Reservoir stimulation operations should be conducted for optimal production performance.

\section{Conclusions}

(1) Larger pressure drop and fluid flow velocity appear at the fracture parts with larger curvatures.

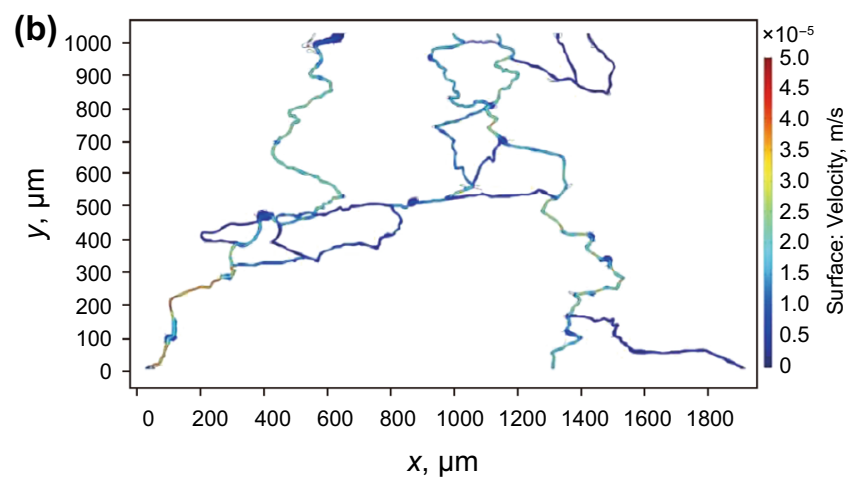

Fig. 15 Distributions of $x$-direction (left) and $y$-direction (right) effective cracks of the $0^{\circ}-180^{\circ}$ cross section plane 

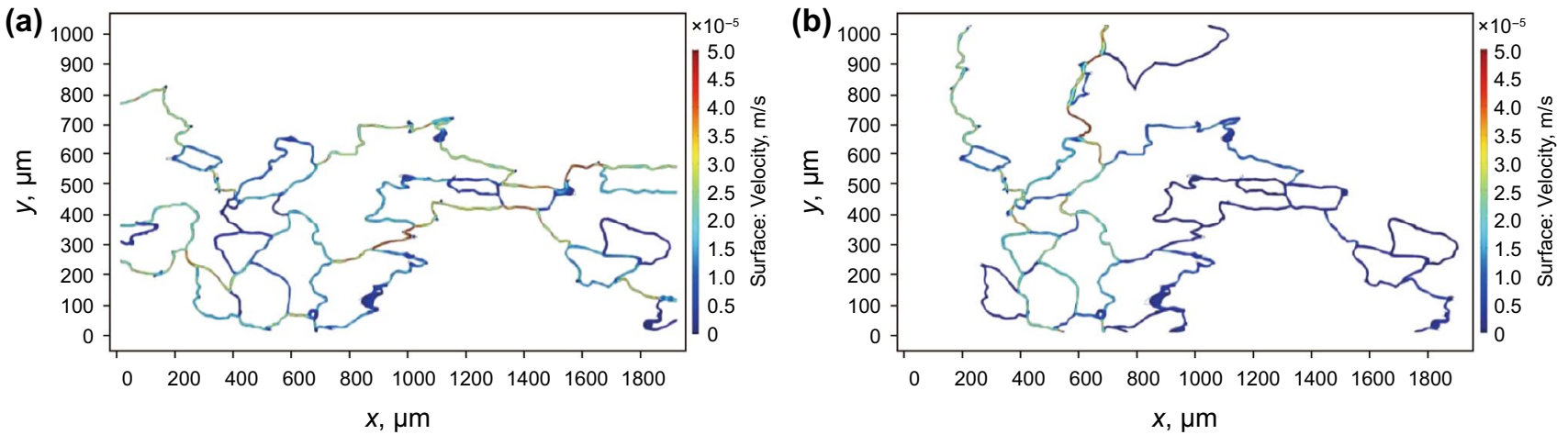

Fig. 16 Distributions of $x$-direction (left) and $y$-direction (right) effective cracks of the $90^{\circ}-270^{\circ}$ cross section plane

Table 3 Image processing results and fracture effectiveness in the $x$ (axial) direction of different cross section planes

\begin{tabular}{|c|c|c|c|c|c|}
\hline $\begin{array}{l}\text { Plane direction, } \\
\text { degree }\end{array}$ & Total fractures & $\begin{array}{l}\text { Number of effective } \\
\text { fractures }\end{array}$ & $\begin{array}{l}\text { Fracture effec- } \\
\text { tiveness }\end{array}$ & Average fracture effectiveness & Standard deviation \\
\hline $0-180$ & 92,773 & 38,454 & 0.4145 & 0.4387 & 0.0804 \\
\hline 15-195 & 72,878 & 24,154 & 0.3314 & & \\
\hline $30-210$ & 72,223 & 25,558 & 0.3539 & & \\
\hline $45-225$ & 66,383 & 24,168 & 0.3641 & & \\
\hline $60-240$ & 75,368 & 35,055 & 0.4651 & & \\
\hline $75-255$ & 65,808 & 28,367 & 0.4311 & & \\
\hline $90-270$ & 98,057 & 63,089 & 0.6434 & & \\
\hline $105-285$ & 60,178 & 24,991 & 0.4153 & & \\
\hline $120-300$ & 56,030 & 25,235 & 0.4504 & & \\
\hline $135-315$ & 70,241 & 29,673 & 0.4224 & & \\
\hline $150-330$ & 80,940 & 35,441 & 0.4379 & & \\
\hline $165-345$ & 63,774 & 34,080 & 0.5344 & & \\
\hline
\end{tabular}

Table 4 Image processing results and fracture effectiveness in the $y$ (radial) direction of different cross section planes

\begin{tabular}{|c|c|c|c|c|c|}
\hline $\begin{array}{l}\text { Plane direction, } \\
\text { degree }\end{array}$ & Total fractures & $\begin{array}{l}\text { Number of effective } \\
\text { fractures }\end{array}$ & $\begin{array}{l}\text { Fracture effec- } \\
\text { tiveness }\end{array}$ & Average fracture effectiveness & Standard deviation \\
\hline $0-180$ & 92,773 & 44,396 & 0.4785 & 0.4806 & 0.0673 \\
\hline $15-195$ & 72,878 & 37,020 & 0.5080 & & \\
\hline $30-210$ & 72,223 & 36,517 & 0.5056 & & \\
\hline $45-225$ & 66,383 & 25,330 & 0.3816 & & \\
\hline $60-240$ & 75,368 & 40,803 & 0.5414 & & \\
\hline $75-255$ & 65,808 & 29,689 & 0.4511 & & \\
\hline $90-270$ & 98,057 & 61,282 & 0.6250 & & \\
\hline $105-285$ & 60,178 & 25,221 & 0.4191 & & \\
\hline $120-300$ & 56,030 & 20,879 & 0.3726 & & \\
\hline $135-315$ & 70,241 & 35,283 & 0.5023 & & \\
\hline $150-330$ & 80,940 & 37,454 & 0.4627 & & \\
\hline $165-345$ & 63,774 & 33,116 & 0.5193 & & \\
\hline
\end{tabular}


(2) The simulation results indicate incomplete development of micro-cracks in the investigated tight sandstone core sample. The connectivity between micro-cracks is poor, resulting in zero flow velocity in many micro-cracks and low fracture effectiveness.

(3) The digital core approach using micro-CT scanning and numerical simulation can readily obtain permeability at any interested direction without complex coring process, overcoming the limitations of conventional experimental approach in measuring directional permeability.

(4) The digital coring approach proposed in this paper provides a method to quantify fracture effectiveness, important information for reservoir evaluation.

Acknowledgements This work was financially supported by the National Natural Science Foundation of China (Grant No. 41972138) and the Technology Major Project of China (Grant No. ZD2019-183007, 2016ZX05002-002).

Open Access This article is licensed under a Creative Commons Attribution 4.0 International License, which permits use, sharing, adaptation, distribution and reproduction in any medium or format, as long as you give appropriate credit to the original author(s) and the source, provide a link to the Creative Commons licence, and indicate if changes were made. The images or other third party material in this article are included in the article's Creative Commons licence, unless indicated otherwise in a credit line to the material. If material is not included in the article's Creative Commons licence and your intended use is not permitted by statutory regulation or exceeds the permitted use, you will need to obtain permission directly from the copyright holder. To view a copy of this licence, visit http://creativecommons.org/licenses/by/4.0/.

\section{References}

An SY, Yao J, Yang YF, et al. Influence of pore structure parameters on flow characteristics based on a digital rock and the pore network model. J Nat Gas Sci Eng. 2016;31:156-63. https://doi. org/10.1016/j.jngse.2016.03.009.

Babchin AJ, Nasr TN. Analytical model for the capillary pressure gradient in oil-water-rock system. Trans Porous Med. 2006;65(2):359-62. https://doi.org/10.1007/s11242-005-6082-7.

Lebourg T, Riss J, Pirard E. Influence of morphological characteristics of heterogeneous moraine Formations on their mechanical behaviour using image and statistical analysis. Eng Geol. 2004;73(12):37-50. https://doi.org/10.1016/j.enggeo.2003.11.004.

Li J, Liu Y, Gao Y, et al. Pore-scale study of the pressure-sensitive effect of sandstone and its influence on multiphase flows. Pet Sci. 2019a;16:382-95. https://doi.org/10.1007/s12182-018-0266-6.

Li P, Jia CZ, Jin ZJ, et al. The characteristics of movable fluid in the Triassic lacustrine tight oil reservoir: a case study of the Chang 7 member of Xin' anbian Block, Ordos Basin, China. Mar Pet Geol. 2019b;102:126-37. https://doi.org/10.1016/j.marpetgeo.2018.11.019.

Lv WF, Chen SY, Gao Y, et al. Evaluating seepage radius of tight oil reservoir using digital core modeling approach. J Pet Sci Eng. 2019;178:609-15. https://doi.org/10.1016/j.petrol.2019.03.072.

Mutailipu M, Liu Y, Wu BH, et al. Gas-water two phase flow simulation based on pore network model for reservoir rocks. Energy Procedia. 2017;142:3214-9. https://doi.org/10.1016/j.egypr o.2017.12.493.
Nishank S, Amie H, Ronny H, et al. Rock properties from micro-CT images: digital rock transforms for resolution, pore volume, and field of view. Adv Water Resour. 2019;134:103419. https://doi. org/10.1016/j.advwatres.2019.103419.

Ren XX, Li AF, Fu SS, et al. Experimental study on the oil-water relative permeability relationship for tight sandstone considering the nonlinear seepage characteristics. J Pet Sci Eng. 2018;161:409_ 16. https://doi.org/10.1016/j.petrol.2017.12.003.

Sadegh K, Pejman T. Segmentation of digital rock images using deep convolutional autoencoder networks. Comput Geosci. 2019;126:142-50. https://doi.org/10.1016/j.cageo.2019.02.003.

Shan PF, Lai XP. Mesoscopic structure PFC 2D model of soil rock mixture based on digital image. J Vis Commun Image R. 2019;28:407-15. https://doi.org/10.1016/j.jvcir.2018.12.015.

Song Z, Zhou QY. Micro-scale granite permeability estimation based on digital image analysis. J Pet Sci Eng. 2019;180:176-85. https ://doi.org/10.1016/j.petrol.2019.05.037.

Starnoni M, Pokrajac D, Neilson JE. Computation of fluid flow and pore-space properties estimation on micro-CT images of rock samples. Comput Geosci. 2017;106:118-29. https://doi. org/10.1016/j.cageo.2017.06.009.

Sun HF, Belhaj H, Tao G, et al. Rock properties evaluation for carbonate reservoir characterization with multi-scale digital rock images. J Pet Sci Eng. 2019;175:654-64. https://doi.org/10.1016/j.petro 1.2018.12.075.

Tang J, Zhang CG, Xin Y. A fracture evaluation by acoustic logging technology in oil-based mud: a case from tight sandstone reservoirs in Keshen area of Kuqa Depression, Tarim Basin, NW China. Pet Explor Dev. 2017;44(3):418-27. https://doi. org/10.1016/S1876-3804(17)30048-4.

Wang G, Qin XJ, Shen JN, et al. Quantitative analysis of microscopic structure and gas seepage characteristics of low-rank coal based on CT three-dimensional reconstruction of CT images and fractal theory. Fuel. 2019a;256:115900. https://doi.org/10.1016/j. fuel.2019.115900.

Wang G, Shen JN, Liu SM, et al. Three-dimensional modeling and analysis of macro-pore structure of coal using combined $\mathrm{X}$-ray CT imaging and fractal theory. Int J Rock Mech Min. 2019b;123:104082. https://doi.org/10.1016/j.ijrmms.2019.10408 2.

Wang QC, Chen DX, Gao XZ, et al. Microscopic pore structures of tight sandstone reservoirs and their diagenetic controls: a case study of the Upper Triassic Xujiahe Formation of the Western Sichuan Depression, China. Mar Pet Geol. 2020;113:104119. https://doi.org/10.1016/j.marpetgeo.2019.104119.

Yin S, Han C, Wu ZH, et al. Developmental characteristics, influencing factors and prediction of fractures for a tight gas sandstone in a gentle structural area of the Ordos Basin, China. J Nat Gas Sci Eng. 2019;72:103032. https://doi.org/10.1016/j.jngse.2019.10303 2.

Zhang F, Nagel NB, Lee B, et al. The influence of fracture network connectivity on hydraulic fracture effectiveness and microseismcity generation. In: 47th US rock mechanics/geomechanics symposium, 23-26 June, San Francisco, CA, USA, 2013.

Zhang F, Mack M. Integrating fully coupled geomechanical modeling with microseismicity for the analysis of refracturing treatment. J Nat Gas Sci Eng. 2017;46:16-25. https://doi.org/10.1016/j.jngse 2017.07.008.

Zhang LH, Shan BH, Zhao YL, et al. Review of micro seepage mechanisms in shale gas reservoirs. Int J Heat Mass Tran. 2019;139:14479. https://doi.org/10.1016/j.ijheatmasstransfer.2019.04.141.

Zhao XY, Hu XY, Zeng LB, et al. Evaluation on the effectiveness of natural fractures in Changxing Fm reef-flat facies reservoirs, Yuanba area, Sichuan Basin. Nat Gas Ind B. 2017;4(4):239-48. https://doi.org/10.1016/j.ngib.2017.08.006. 